Article

\title{
Impact of Load Variation on the Accuracy of Gait Recognition from Surface EMG Signals
}

\author{
Xianfu Zhang ${ }^{1,2, *}$, Shouqian Sun ${ }^{1}$, Chao $\mathrm{Li}^{1}{ }^{1}$ and Zhichuan Tang ${ }^{3}$ (D) \\ 1 College of Computer Science and Technology, Zhejiang University, Hangzhou 310027, China; \\ ssq@zju.edu.cn (S.S.); superli@zju.edu.cn (C.L.) \\ 2 School of Jewelry and Art Design, Wuzhou University, Wuzhou 543002, China \\ 3 Industrial Design Institute, Zhejiang University of Technology, Hangzhou 310023, China; ttzzcc@zju.edu.cn \\ * Correspondence: zhangxianfu@zju.edu.cn
}

Received: 26 July 2018; Accepted: 22 August 2018; Published: 25 August 2018

\begin{abstract}
As lower-limb exoskeleton and prostheses are developed to become smarter and to deploy man-machine collaboration, accurate gait recognition is crucial, as it contributes to the realization of real-time control. Many researchers choose surface electromyogram (sEMG) signals to recognize the gait and control the lower-limb exoskeleton (or prostheses). However, several factors still affect its applicability, of which variation in the loads is an essential one. This study aims to (1) investigate the effect of load variation on gait recognition; and to (2) discuss whether a lower-limb exoskeleton control system trained by sEMG from different loads works well in multi-load applications. In our experiment, 10 male college students were selected to walk on a treadmill at three different speeds (V3 $=3 \mathrm{~km} / \mathrm{h}, \mathrm{V} 5=5 \mathrm{~km} / \mathrm{h}$, and V7 $=7 \mathrm{~km} / \mathrm{h}$ ) with four different loads ( $\mathrm{L} 0=0$, $\mathrm{L} 20=20 \%, \mathrm{~L} 30=30 \%, \mathrm{~L} 40=40 \%$ of body weight, respectively), and 50 gait cycles were performed. Back propagation neural networks (BPNNs) were used for gait recognition, and a support vector machine (SVM) and k-nearest neighbor (k-NN) were used for comparison. The result showed that (1) load variation has significant effects on the accuracy of gait recognition $(p<0.05)$ under the three speeds when the loads range in L0, L20, L30, or L40, but no significant impact is found when the loads range in L0, L20, or L30. The least significant difference (LSD) post hoc, which can explore all possible pair-wise comparisons of means that comprise a factor using the equivalent of multiple $t$-tests, reveals that there is a significant difference between the L40 load and the other three loads (L0, L20, L30), but no significant difference was found among the L0, L20, and L30 loads. The total mean accuracy of gait recognition of the intra-loads and inter-loads was $91.81 \%$, and $69.42 \%$, respectively. (2) When the training data was taken from more types of loads, a higher accuracy in gait recognition was obtained at each speed, and the statistical analysis shows that there was a substantial influence for the kinds of loads in the training set on the gait recognition accuracy $(p<0.001)$. It can be concluded that an exoskeleton (or prosthesis) control system that is trained in a single load or the parts of loads is insufficient in the face of multi-load applications.
\end{abstract}

Keywords: sEMG; load variation; gait recognition; lower-limb exoskeletons

\section{Instruction}

Lower-limb exoskeletons and prostheses allow stroke patients and lower limb hemiplegia patients much greater convenience, especially in terms of improving their stability for walking on flat ground [1]. With in-depth studies, lower-limb exoskeletons and prostheses have been developed in the direction of smarter man-machine collaborations, which enhance the ability of man-machine coordination, to improve the rehabilitative effect by detecting the wearer's intention of motion [2,3]. This requires adjustment of the control signal input for the lower-limb exoskeleton and prostheses according to 
real-time gait information. Thus, accurate gait recognition is crucial, as it contributes to the realization of real-time control. For the gait phases in gait recognition, the existing division includes five phases [4], seven phases [5], and eight phases in a gait cycle [6]. In our study, we adopted the mainstream five-phase division in a gait cycle, which consists of pre-stance, mid-stance, terminal stance, pre-swing, and terminal swing per cycle.

Many researchers have adopted surface electromyogram (sEMG) signals to recognize gait for the control of lower-limb exoskeletons (or prostheses) [7]. Due to their capability for of revealing the inner workings of the human motor nerve system, sEMG signals can directly reflect the subject's intended activity, and the motor function instruction information of the neuromuscular system [8]. In addition to the convenience and non-invasive nature of the signal acquisition, sEMG signals are also widely used as a neural control source for human-machine interaction, such as the control of lower-limb exoskeletons (or prostheses). Joshi et al. [5] employed a Bayesian information criterion (BIC) and a linear discriminant analysis (LDA) classification algorithm to deal with lower-limb sEMG signals for gait recognition by determining the boundaries of adjacent gait phases in seven gait phases (loading response, mid-stance, terminal stance, pre-swing, initial swing, mid-swing, and terminal swing). Li et al. [4] employed support vector machines (SVMs) and lower-limb sEMG to demonstrate that both individual differences and sample sizes have an influence on gait classification accuracy. Yang et al. [9] took sEMGs from a disabled thigh as control signals to drive artificial lower limbs based on support vector machines and achieved conversion from standing to walking. Wu et al. [10] proposed a multiple classification algorithm for lower-limb movement recognition based on a simplified support vector machine, and successfully identified the movements of standing, walking, and going up and down the stairs.

The works mentioned above focus mainly on increasing the accuracy of gait phase recognition via sEMG data collection via sensors, effective preprocessing of data, reasonable extraction of feature vectors and algorithm optimization, etc. Although these methods may achieve an ideal accuracy of gait recognition of up to $95 \%$ in laboratory conditions, the results are not so encouraging in real-time environments. There are several practical limitations for sEMGs for use in gait recognition for the control of exoskeletons and prostheses. Lee et al. [8] demonstrated that performance in gait recognition is not guaranteed as a result of the influence of the variable environmental factors. The most common variable factors include different lower-limb positions [11,12], sEMG signal instability [13], displacement of the electrode [14], and so on; moreover, load variation is a problem that cannot be ignored. Some scholars have studied the effects of different loads on pattern recognition based on electromyography. For instance, Al-Timemy et al. [15] revealed that the load variation can affect the recognition accuracy (about $60 \%$ difference between best and worst accuracy) for the same control system, based on sEMG. Scheme et al. [16] demonstrated that the results from pattern recognition (error rates ranging from $8 \%$ to $19 \%$ ) when the training and testing data are from the same level of force, were much better than the results (error rates ranging from $32 \%$ to $44 \%$ ) when they were from different levels of force. Tang et al. [17] studied the impact of different loads on the prediction accuracy of a joint angle based on sEMG, and they remarkably improved the estimation performance through multi-sensor fusion. Despite the brilliant results, this does not mean that the same conclusion certainly exists for gait recognition based on sEMG when the loads vary. In addition, few researchers have studied the influence of load variation on the accuracy of gait recognition from sEMG, so this area is still not well understood.

To study this issue in-depth, we performed our experiment under four different loads at three speeds, and we employed back propagation neural networks (BPNNs) to obtain accurate gait recognition for every trial, and then we used the statistics to analyze the fluctuations in the accuracy of gait recognition with the load change at each speed. SVM and k-NN were used to compare the results, to clarify whether the result was caused by the algorithm or the load variation. Based on the experiment, we studied the impact of intra-loads and inter-loads on gait recognition accuracy, and we simulated the potential load state that is faced by people in real-time environments by combining 
the data from different loads to demonstrate (1) whether the load variation has a significant influence on gait recognition; and (2) whether an exoskeleton (or prosthesis) control system trained by sEMG signals from single or parts of loads still works well under multi-load conditions.

The remainder of the paper is organized as follows. Section 2 describes in detail the processes and methods of the experiment. Section 3 presents the results of the experiment and analyzes the data, including the effect of load variation on the accuracy of gait recognition, the comparison results for the three algorithms, an analysis of differences between intra-loads and inter-loads in gait recognition accuracy, and an analysis of the influence of load variation on gait recognition in a simulated real-time environment. Section 4 discusses the reasons for our results, and Section 5 presents our conclusions.

\section{Experimental Method}

\subsection{Subjects}

In our study, 10 male subjects (age range: $24-28$ years old, height range: $173.0-176.0 \mathrm{~cm}$, weight range: $62.1-71 \mathrm{~kg}$ ) without any illness in their musculoskeletal and nervous systems, were selected from graduate students who were skilled in using a treadmill (Brother WL-332). Before the experiment, they were not allowed to take part in physical exercise to avoid complications arising from fatigue. The experiment was approved by the local human ethics committee of Zhejiang University, and all the volunteers provided us with informed consents in advance.

\subsection{Experimental Procedure}

According to the different effects that the lower limb muscles exert in the gait cycle, we selected the four thigh muscles to acquire sEMG signals as described in [4], that is, the tensor fasciae latae (tensor) and the semitendinosus (semitend), which are more active during the stance phase than the swing phase, as well as the adductor longus (adductor) and the vastus medialis (vmo), which play very important roles in the swing phase $[4,18]$, as shown in Figure 1.

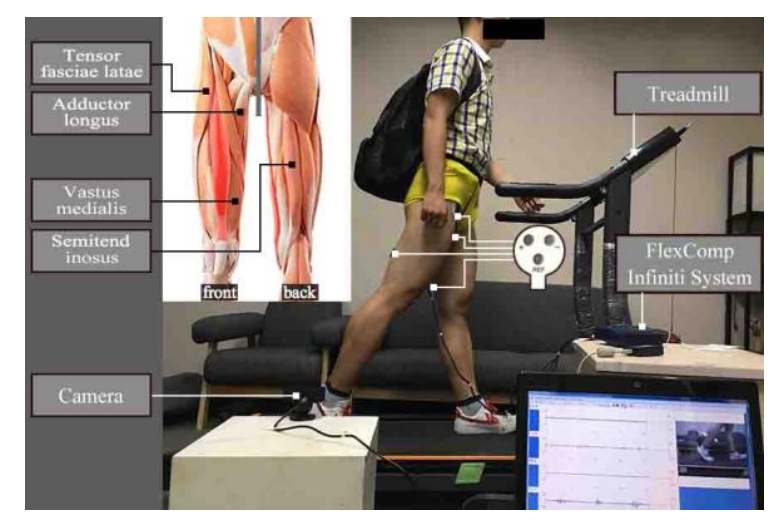

Figure 1. The experimental equipment and the placement of the four-surface electromyogram (sEMG) electrodes.

Before each experiment, the subjects were asked to wear shorts and white shoes in order to show a sharp contrast with the treadmill, for the observation of the gait phase. Their anthropometric measurements—including age, height, and weight—-were taken in advance, and then they were asked to become familiar with the experimental procedure, treadmill, and other equipment which would be used in the experiment. Alcohol was used to clean the skin around the target muscle after any hair was shaved off, and electrolyte gel was utilized to reduce any interference produced by the skin, and to improve the contact stability and the electrical conductivity of the electrodes [19].

Four electrode groups, each electrode group consisting of three $\mathrm{Ag} / \mathrm{AgCl}$ electrodes (a positive electrode, a negative electrode, and a reference electrode), were attached to the four target muscles, and 
a line between the positive and negative electrodes was made exactly in accordance with the direction of the muscle fibers on the midline of the muscle belly [20]. We then marked the exact location on the skin with a marking pen to make sure that the electrodes could be attached to the same location in every experiment, and new electrodes were employed in each trial. Medical bandages were used to fix the wires on the thigh, to reduce the disturbance of shaking wires on the sEMG signal, and the bandage was fixed so that it was not too tight, so as not to interfere with action of the muscle, nor too loose so as to not dislodge the wire.

After all the equipment was connected as shown in Figure 1, and all signals were normal, every subject was required to perform 50 continuous gait cycles on the treadmill at a constant speed, under four different loads of L0 $=0$ (no load), L20 $=20 \%, \mathrm{~L} 30=30 \%$, and L40 $=40 \%$ of body weight, respectively [21], at speeds of $\mathrm{V} 3=3 \mathrm{~km} / \mathrm{h}, \mathrm{V} 5=5 \mathrm{~km} / \mathrm{h}$, and $\mathrm{V} 7=7 \mathrm{~km} / \mathrm{h}$ [22]. This resulted in 6000 data sets ( 10 subjects $\times 50$ gait cycles $\times 4$ loads $\times 3$ speeds) produced in the experiment. The 50 gait cycles acted as the dividing line to show whether gait cycle number had a significant impact on gait recognition accuracy [4].

In the experiment, a traditional commercial backpack (U.S. Polo Assn Sport Backpack, Colfax, LA, USA) without a sternum strap or a hip-loading belt was used to carry the load, to suggest a more convincing universal applicability. The backpack load was increased in increments of $0.1,0.2,0.5,1$, 2 , and $5 \mathrm{~kg}$ to match the 20,30 , and $40 \%$ of the subject's body weight, and the load was distributed symmetrically on both sides of the spine inside the backpack. The shoulder straps were then adjusted to ensure placement of the backpack above the hips at the low back [23].

For each trial, when the subjects thought that they were walking steadily on the treadmill at the required speed, they sent instructions to the staff to collect signals. After each trial, the subjects were required to have a $10 \mathrm{~min}$ rest to recover from the fatigue caused by the exertion to avoid negative effects on the next experiment.

\subsection{Data Acquisition}

The MyoScan sensor was used to collect the sEMG signals (up to $1600 \mu \mathrm{V}$ ) from the subject's four target muscles. The sEMG was filtered in the range of $10-400 \mathrm{~Hz}$, and a $50 \mathrm{~Hz}$ notch filter was set to remove the line interference. The collected raw sEMG signals then were amplified and transmitted to a computer for data analysis through the FlexComp Infiniti System (Thought Technology Ltd., Montreal, QC, Canada), which contains a digital sEMG system with 10 channels and a video collection system [19]. It can ensure the synchronism of the sEMG signal and the video data. The sampling rate of sEMG signals were $2048 \mathrm{~Hz}$. The video data was acquired simultaneously at a resolution of 60 frames per second, using an external high definition high speed camera when each trial was performed. The camera was placed perpendicular to the walking direction of the treadmill, and the distance between the camera and the treadmill was adjusted to ensure that the entire running belt of the treadmill was in the camera's field of view [24].

\subsection{Signal Preprocessing}

All of the sEMG signals and videos were processed offline. Each of the videos was segmented into a single gait cycle and then further divided into five gait phases (pre-stance, mid-stance, terminal stance, pre-swing, terminal swing) by identifying the demarcation point of the heel strike and toe off of both feet by an experienced observer [25]. The segmented video was then used as a reference for the five gait phases that were labeled on the corresponding sEMG signal [26].

The overlapped windowing technique [7] was employed to segment the labeled sEMG signal for feature extraction in this paper (see Section 2.5), and we set $20 \mathrm{~ms}$ windows for feature extraction, with one window being overlapped by $10 \mathrm{~ms}$, i.e., half of the last window. 


\subsection{Feature Extraction}

The main purpose of feature extraction was to reduce the dimension of the original surface sEMG signal to reduce the complexity of the pattern recognition and classification, and then to improve the efficiency. Therefore, it was crucial to extract features with good distinction, low complexity, and high efficiency [27]. At present, the main feature extraction methods were time domain, frequency domain, and time-frequency domain analysis. In the field of human-computer interaction, time domain features are widely used, due to their excellent signal classification performance and lower computational complexity in a low-noise environment. [28,29]. In this paper, we chose the root mean square (RMS) [12] and integrated EMG (iEMG) as the input features, where the RMS reflected the amplitude variation of the surface EMG signals on the time scale, and the $i E M G$ referred to the total area of the sEMG signal curve after being rectified and filtered: this reflected the changes in the sEMG signal strength in the time dimension [29], and they can be expressed as in Equations (1) and (2).

$$
\begin{aligned}
& R M S=\sqrt{\frac{1}{N} \sum_{i=1}^{N} X_{i}^{2}} \\
& i E M G=\frac{1}{N} \sum_{i=1}^{N}\left|X_{i}\right|
\end{aligned}
$$

where $X_{i}$ is the $i$ th sEMG time samples sequence. $i=1,2 \ldots, N$. $N$ is the number of sampling points in each analysis window.

Thus, the input vector $Z$ is constructed:

$Z_{i}=\left\{R M S_{i 1}, i E M G_{i 1}, R M S_{i 2}, i E M G_{i 2}, R M S_{i 3}, i E M G_{i 3}, \ldots, R M S_{i j}, i E M G_{i j}\right\}$, in which, $i=1,2,3$, $\ldots, K, K$ is the number of analysis windows; $j=1,2,3, \ldots, M$, and $M$ is the number of the channels.

In our experiment, four muscles were selected for collection of the sEMG signals, which meant that there would be eight features in each time window to be used as input vectors. The outputs were composed of five gait phases (pre-stance, mid-stance, terminal stance, pre-swing, and terminal swing).

\subsection{Classification Methods}

Two steps were generally followed in this stage: (a) model training, where BPNN, SVM, and k-NN were trained for the gait phase class, and (b) testing, and then the accuracy of the gait recognition was obtained. For both training and testing steps, eight-dimensional feature vectors (four channels, with each channel including two types of features, RMS and $i E M G$ ) from each segmented sEMG in the previous stage were used as inputs for the three training models.

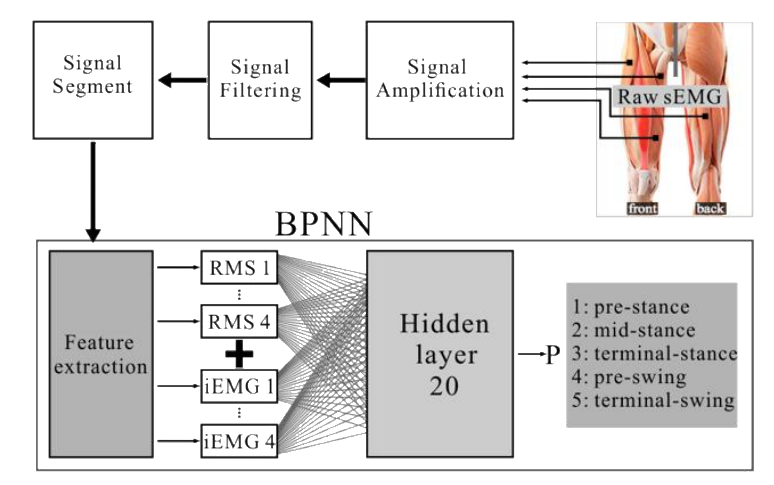

Figure 2. Schematic structure of the BPNN, including 20 nodes in the hidden layer, where eight features were extracted as input from the segmented sEMG signal after amplification, filtering, and segmenting, and the output layer consisted of a single node (phase). 
A back-propagation neural network (BPNN) is a multilayer feedforward network that is trained by error back-propagation. The basic idea is a gradient descent method, which uses the gradient search technique to achieve the minimum error mean variance of the actual output value and the expected output value of the network. Because of its remarkable performance in nonlinear mapping and flexible network structure, BPNN has been widely used for pattern recognition and classification based on sEMG signals $[17,30]$. In this study, we constructed a three-layer BPNN, and set 20 nodes in the hidden layer; the classification scheme is shown in Figure 2. The input layer consisted of eight nodes (four channels of sEMG signals $\times$ two features) from the $20 \mathrm{~ms}$ long analysis windows, and the output layer consisted of a single node, which was the gait phase of the corresponding analysis window.

The support vector machine (SVM) is a machine learning method based on statistics. The key is to choose a reasonable kernel function with which the vector is mapped to a higher dimensional space, in order to determine the nonlinear relationship between an input and output. The selection of the appropriate kernel function has greater significance when the data cannot be linearly separated. Oskoei et al. [31] demonstrated a good classification result for linearly inseparable data using a library for support vector machines (LIB-SVM) to develop the SVM classifier. In our study, the Gauss radial basis function (RBF) was employed to build the SVM model because of its good adaptability to the size of the data, and its good robustness [31,32]. A cross-validation was applied to structure the classification model in the training phase.

The k-NN classification algorithm is one of the simplest methods of the classification techniques. The core idea of $\mathrm{k}-\mathrm{NN}$ is that if most of the k-nearest neighbor samples of a sample belong to a category in the feature space, the sample belongs to the category too, and accordingly it possesses the characteristics of this type of sample. Despite its simplicity, the k-NN method had been used successfully in a wide variety of classification problems [27,33]. In our study, we used the Euclidean distance in the feature space to evaluate which k-training samples were the nearest neighbors [33]. Because 50 gait recycles were performed at each trial for every subject, we compared several $\mathrm{k}$ values (from 2 to 10), and finally chose $\mathrm{k}=5$ as the proper value for the k-NN model, for its good classification performance and efficiency.

\subsection{Data Analysis}

To study the effect of load variation on gait recognition, the BPNN models were trained and tested with the sEMG data from four different loads under three different speeds (12 trials for each subject and 120 trials in total). The inputs of the BPNNs models were the RMS and $i E M G$ of the four muscles, and the outputs were the five gait phases. In each experiment, $80 \%$ of the data was randomly selected for use in training the BPNN models, and the remaining $20 \%$ of the data was used to test the models. In the training phase of BPNNs, a 10-fold cross-validation method was employed to test the accuracy of the model, in which the selected data was randomly divided into 10 parts, with nine parts being used to train the model, and the remaining part being used for validation [17,32]. The average recognition accuracy of a single trial was acquired by averaging the accuracy of the five gait phases of the trial, which were gained separately. For a comparison, the SVM and k-NNs models were used to deal with the same data.

As for the accuracy of the gait recognition, univariate analysis of variance (UNIANOVA) was employed to analyze the effect of the load variation and speed on the accuracy of the gait recognition. If the effect was significant, the least significant difference (LSD) post hoc test was carried out to fully explore this effect. All data were analyzed by SPSS version 24.0 for Mac (SPSS Inc., Chicago, IL, USA), and the statistical significance was determined at $p<0.05$.

\section{Results}

All the data from the four muscles of the 10 subjects was processed, and data from a $2 \mathrm{~s}$ long sample (sEMG, RMS, IEMG) from a subject under L20 at V5 is shown in Figure 3. This revealed that the four muscles played different roles in the gait cycles. Of these, the tensor fasciae latae and the 
semitendinosus were more active in the stance phase; the adductor longus and the vastus medialis played a more important role in the swing phase.
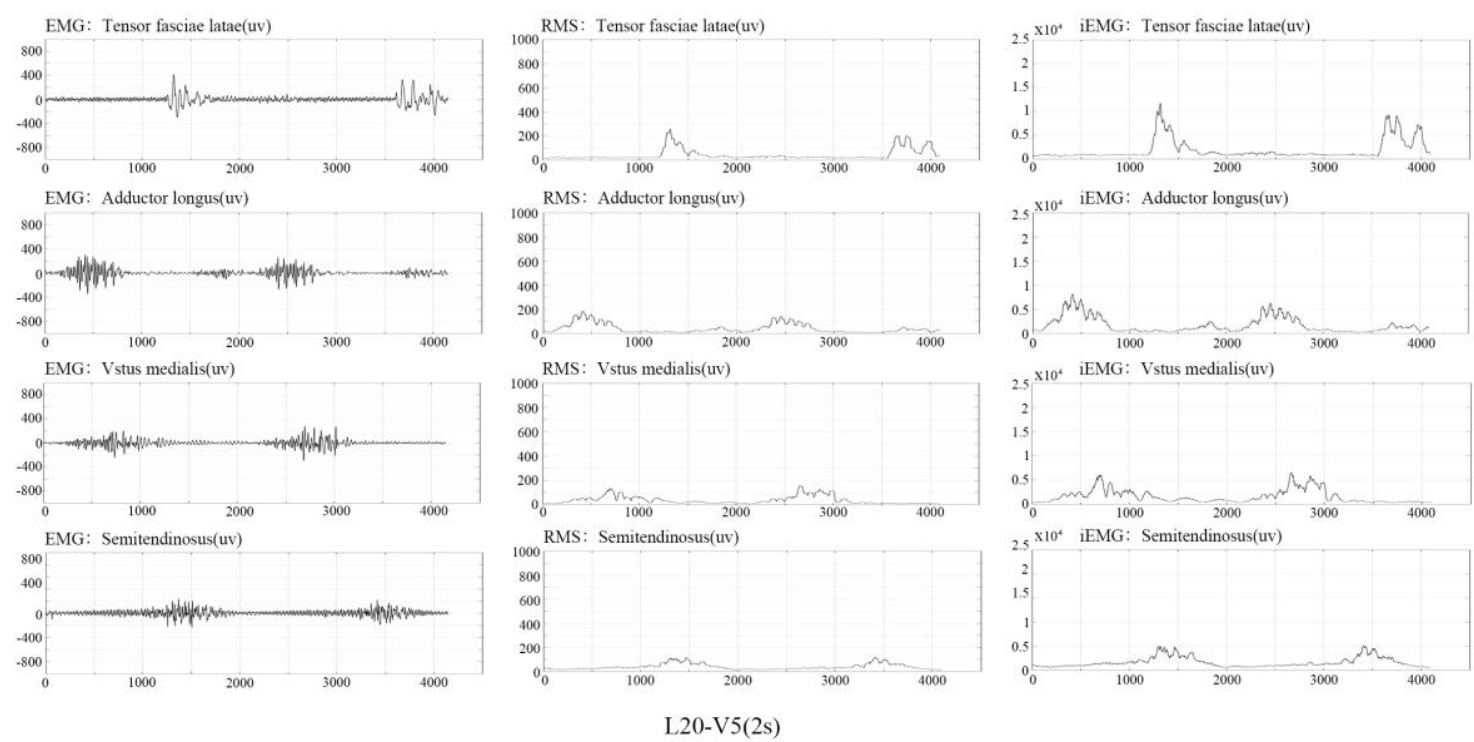

Figure 3. Raw sEMG, RMS, and $i E M G$ for the four muscles from one subject, with L20 at V5.

\subsection{Accuracies of Five Gait Phases under Different Loads}

Four different BPNN models were trained at each speed. As we can see in Table 1, the total average accuracies of gait recognition (total Avg) under the four loads at three speeds were very different. The highest value was $94.25 \%$ at $\mathrm{V} 5$, followed by $92.55 \%$ at $\mathrm{V} 7$, and the minimum value was only $88.61 \%$ at V3. There was also a significant difference in load when the highest accuracy of gait recognition appeared at each speed. At V3, the highest accuracy of gait recognition (91.94\%) occurred when the load was L30. At V5, the highest value (97.56\%) appeared when the load was L20, which was the highest among the four loads and three speeds. When the speed was V7, the highest value $(94.62 \%)$ appeared when the load was L0 (no load). At the same time, when the load was increased to L40, the gait recognition accuracy was the lowest among the three speeds $(83.05 \%$ at V3, $91.18 \%$ at V5, and $89.7 \%$ at $\mathrm{V} 7$, respectively).

Table 1. Accuracy of recognition of the five gait phases (\%), the accuracy of the gait recognition (\%) under each load and speed, and the total average accuracy of the gait recognition (Total Avg) (\%) among the four loads under each speed.

\begin{tabular}{|c|c|c|c|c|c|c|c|c|c|c|c|c|}
\hline \multirow{2}{*}{$\begin{array}{l}\text { Speed } \\
\text { Load }\end{array}$} & \multicolumn{4}{|c|}{ V3 } & \multicolumn{3}{|c|}{ V5 } & \multicolumn{5}{|c|}{ V7 } \\
\hline & L0 & L20 & L30 & L40 & L0 & L20 & L30 & L40 & 0 & L20 & L30 & L40 \\
\hline Pre-stance & 89.25 & 96.00 & 84.81 & 80.89 & 97.69 & 96.61 & 86.33 & 83.57 & 92.21 & 90.35 & 94.39 & 89.51 \\
\hline Mid-stance & 98.71 & 90.40 & 98.25 & 87.18 & 94.67 & 99.14 & 99.49 & 98.17 & 99.35 & 99.03 & 98.17 & 98.52 \\
\hline Terminal stance & 91.53 & 95.18 & 95.65 & 88.47 & 86.84 & 95.27 & 95.50 & 91.71 & 89.57 & 88.22 & 86.07 & 88.51 \\
\hline Pre-swing & 80.11 & 72.69 & 88.00 & 86.61 & 97.00 & 98.14 & 92.71 & 95.58 & 94.57 & 92.24 & 89.86 & 94.29 \\
\hline Terminal swing & 94.55 & 88.73 & 92.99 & 72.10 & 98.90 & 98.63 & 92.07 & 86.87 & 97.42 & 98.41 & 92.71 & 80.18 \\
\hline Accuracy & 90.83 & 88.6 & 91.94 & 83.05 & 95.02 & 97.56 & 93.22 & 91.18 & 94.62 & 93.65 & 92.24 & 89.70 \\
\hline Total Avg & \multicolumn{4}{|c|}{88.61} & \multicolumn{4}{|c|}{94.25} & \multicolumn{4}{|c|}{92.55} \\
\hline
\end{tabular}

The bold are the minimum and the maximum of accuracy of gait recognition among four loads at each speed. 
UNIANOVA indicated that the load variation had significant effects on the accuracy of gait recognition $(p<0.05)$ when the loads were in the range of L0, L20, L30, and L40, but no significant impact was found when the loads are in the range of L0, L20, and L30 ( $p>0.05)$. Furthermore, the LSD post hoc test revealed that there was a significant difference between the L40 load and the other three kinds of loads (L0, L20, L30), but no significant differences were found among the three loads (L0, L20, L30).

As a comparison, the SVM and k-NN algorithms were employed to deal with the same data. In general, the results presented in Figure 4 revealed that the gait recognition accuracy was the highest for the BPNN, followed by k-NN, and lowest for the SVM. Moreover, a similar tendency in gait recognition accuracy was found in the results of the three classification algorithms for all trials. Then, the gait recognition accuracy was analyzed by UNIANOVA, and the same conclusion that the load variation had a significant impact on the accuracy of the gait recognition was obtained (k-NN: $p<0.05$; SVM: $p<0.05$ ).

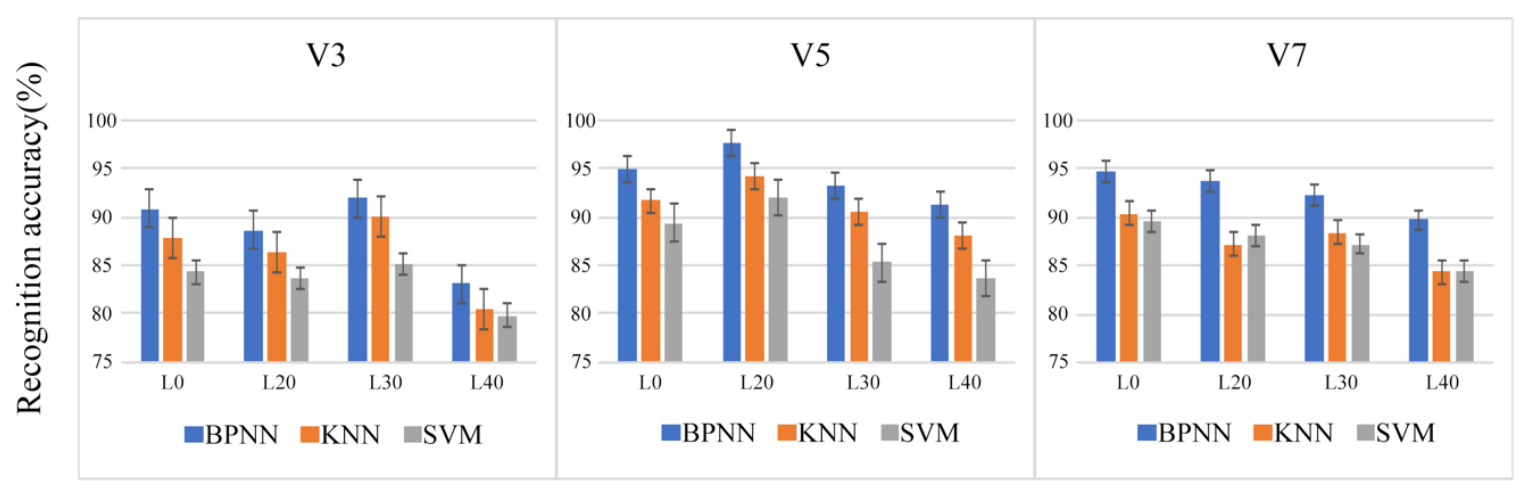

Figure 4. Comparison of three classification algorithms at four different loads under three different speeds.

\subsection{Results of the Confusion Matrix}

Four different BPNNs were trained and tested with the data from each one of the four loads at each speed. The overall results are shown in Figure 5. Each data point in the three matrixes represents an accuracy of gait recognition across all subjects for the designated training and testing loads. In the matrixes, the vertical axis represents the training loads and the horizontal axis represents the test loads. The data shown in the main diagonal comes from the cases where the data that was used for training and testing was obtained from the same load, i.e., intra-load. The other data that was not in the main diagonal came from the cases where the data was used for training and testing as obtained from the different loads, i.e., inter-loads. The $t$-test was employed to demonstrate that there was a significant difference $(p<0.05)$ between the accuracy of the gait recognition from the main diagonal and the gait recognition off the diagonal. This implies the load variation has a great impact on the accuracy of gait recognition. For instance, the results $(83.61,97.56,65.93$, and $67 \%$, respectively) from training with L20 and testing with L0, L20, L30, and L40 at V5, were considerably different. Similar results were found at V3 and V7 under other loads. The poorest results appeared when training with the data from L20, and testing with data from L0, L30 (L20-L0: 52.78\% and L20-L30: 53.62\%, respectively) at V3. The poorest results at V5 came from L20-L30, L0-L30 (65.93\% and 66.23\%, respectively). As for V7, the poorest results came from L20-L30, L20-L40 (62.75\% and 66.48\%, respectively). 


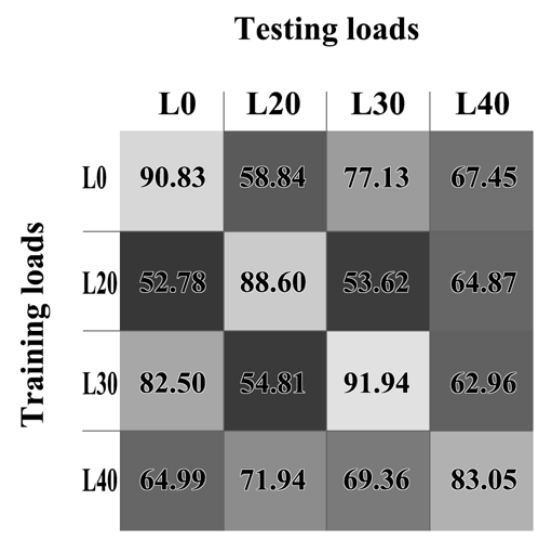

V3
Testing loads

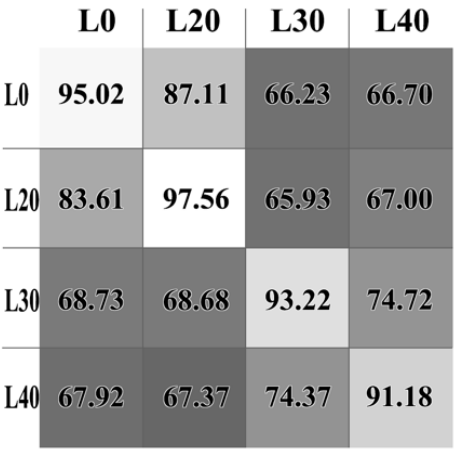

V5
Testing loads

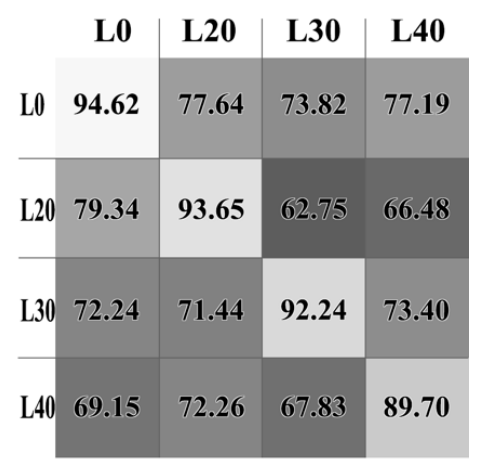

V7

Figure 5. Accuracy of gait recognition (\%) matrices under three speeds. Each value in the matrix represents the accuracy of gait recognition from the specified training loads and testing loads (vertical and horizontal axis, respectively). The whiter color means a higher gait recognition accuracy.

The statistical results of the three recognition accuracy matrices, including recognition accuracy of intra-load, inter-loads, and overall, are shown in Table 2, and expressed as mean $\pm \mathrm{sd}$. The total mean intra-load recognition accuracy was $91.81 \%$, but the total mean overall recognition accuracy was $75.02 \%$, which was far away from the intra-load recognition accuracy. The lowest mean recognition accuracy was the inter-load accuracy, which was only $69.42 \%$. Different speeds were found to have a great impact on the accuracy of gait recognition in Table 2. For instance, the mean overall recognition accuracy at V3 (70.98\%) was substantially different from those at V5 (76.96\%) and V7 (77.11\%).

Table 2. Intra-load, inter-load, and overall accuracy of gait recognition (\%) (mean \pm sd) across the three speeds

\begin{tabular}{ccccc}
\hline & V3 & V5 & V7 & Total \\
\hline Intra-load accuracy (mean \pm sd) & $88.61 \pm 3.96$ & $94.25 \pm 2.71$ & $92.56 \pm 2.14$ & $91.81 \pm 3.69$ \\
Inter-load accuracy (mean \pm sd) & $65.11 \pm 9.30$ & $71.20 \pm 7.47$ & $71.96 \pm 4.86$ & $69.42 \pm 7.86$ \\
Overall accuracy (mean \pm sd) & $70.98 \pm 13.30$ & $76.96 \pm 12.19$ & $77.11 \pm 10.15$ & $75.02 \pm 12.05$ \\
\hline
\end{tabular}

\subsection{Results of the Mixed-Load Evaluation}

In order to study the influence of the training set composed from the data from parts of loads on gait recognition accuracy, 15 training models were constructed for comparison. We gathered all the data together as a test set at each speed (group 3 at V3, group 5 at V5, group 7 at V7), and freely combined the four kinds of data from the four loads as a training set. In all, we obtained 15 different training sets, which were composed of the data from one load (four kinds), two loads (six kinds), three loads (four kinds), or four loads (one kind). The single accuracy of gait recognition and the average accuracy of gait recognition for all the same kinds of loads at each speed are shown in Figure 6. 


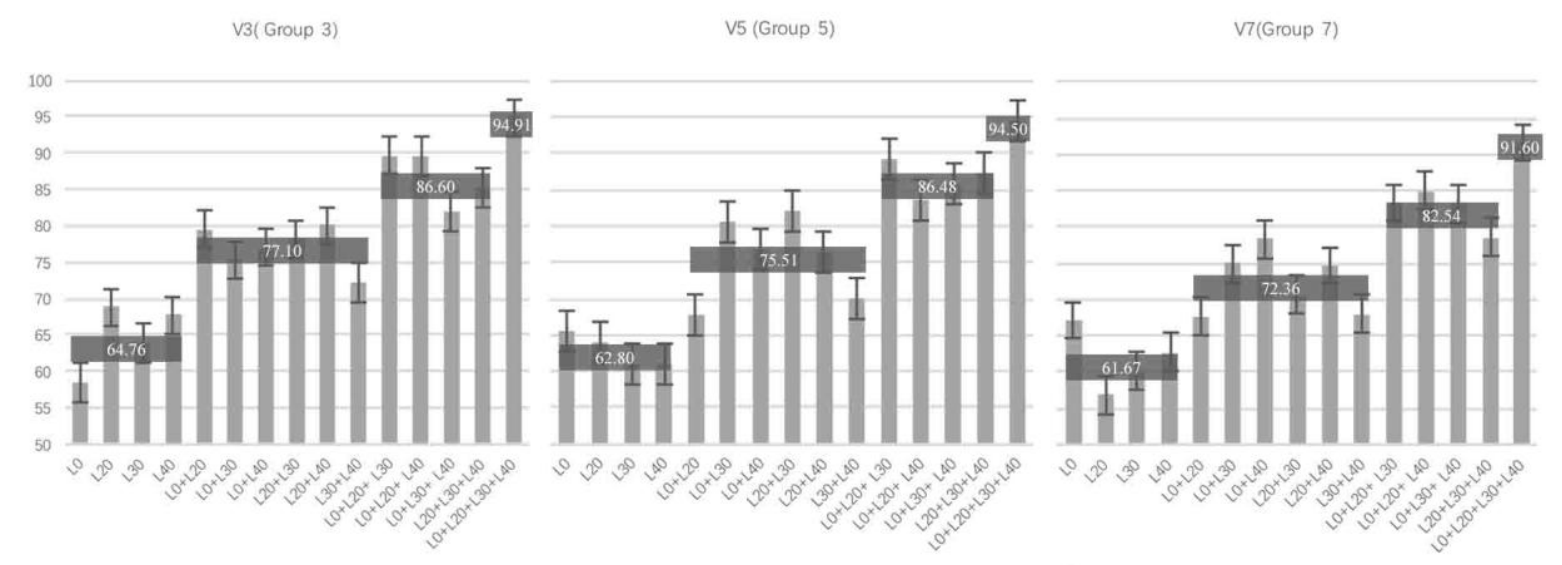

Figure 6. Accuracy of the gait recognition (\%) of the groups at three speeds. The testing data (group 3 at V3, group 5 at V5, group 7 at V7) is from all four loads, the training data is comprised of the data from one load (four kinds), two loads (six kinds), three loads (four kinds), and four loads (one kind) at each speed. The average accuracy of the gait recognition (\%) for all the one load, two loads, three loads, and four loads, at each speed are labeled in the picture.

We noted that when any data set from one load was used as a training set, and the data group at the same speed as the testing set, the average accuracy of the gait recognition was the poorest $(64.76 \%$ at $\mathrm{V} 3,62.80 \%$ at $\mathrm{V} 5$, and $61.67 \%$ at V7). When any of the data from two loads was used as a training set, the average accuracy of the gait recognition was significantly higher $(77.10 \%$ at $\mathrm{V} 3,75.51 \%$ at $\mathrm{V} 5,72.36 \%$ at $\mathrm{V} 7$ ) than that which was obtained when the training set data was from one load. When any of the three data sets from the three loads was used as a training set, the average accuracy of the gait recognition $(86.6 \%$ at $\mathrm{V} 3,86.48 \%$ at $\mathrm{V} 5$, and $82.54 \%$ at $\mathrm{V} 7)$ was more agreeable, which was much higher than the first two. When the training set consisted of the data from all four loads, the accuracy of the gait recognition (94.91 at V3, $94.5 \%$ at $\mathrm{V} 5$, and $91.60 \%$ at $\mathrm{V} 7)$ was the highest in all the cases. The results imply that when the training set data is taken from more types of loads at the same speed, the gait recognition accuracy is better.

\section{Discussion}

In this paper, we investigated the effect of load variation on the accuracy of gait recognition based on sEMG signals. Based on this, we can examine whether an exoskeleton (or prosthesis) control system, which uses myoelectric signals as the control signal, can still work well in the face of multi-load applications when trained as a single load or as parts of loads.

As is shown in Table 1, at each speed, the accuracy of gait recognition under different loads fluctuates obviously (V3: 91.94-83.05\%, V5: 97.56-91.18\%, and V7: 94.62-89.7\%, respectively). The load is closely related to muscle activity [34,35]-i.e., the heavier the load is, the more active the muscle activity is - which more easily leads to muscle fatigue after a period of walking [35], and affects the gait stability and recognition accuracy. Furthermore, due to inertia in walking, a heavier load produces more forward and lateral pull on the human body [23], which increases instability in the gait and reduces the accuracy of gait recognition. Our study also shows that the load variation significantly affects the accuracy of gait recognition when the load is in the L0, L20, L30, and L40 range $(p<0.05)$, but no significant effect is found when the load variation in the L0, L20, and L30 range $(p>0.05)$. One possible reason is that there exists a threshold in the load level [36]. Below this level, the muscle maintains gait stability and the sEMG signals through self-compensation for self-protection [20,35], while when the load level is above the threshold, the load aggravates the gait instability and the sEMG signal, which results in a substantial reduction of the gait recognition accuracy. Some existing studies may provide a certain extent of support for the threshold hypothesis. Simpson et al. [35] revealed that the risk of lower limb injuries may be effectively reduced with a loading of less than $30 \%$ of body 
weight with a backpack, but when the load is increased to $40 \%$, which can cause a later lower limb muscle burst onset.

In order to validate our results, we used the k-NN and SVM algorithms to work with this data separately from the BPNN. The results (Figure 4) revealed that the conclusion where load variation has a significant influence on gait recognition accuracy can still be obtained clearly, although the performances of the two algorithms are slightly weaker than the BPNN overall.

To test whether an exoskeleton can play a strong role when faced with various loads in real-time environments, it is possible to examine whether the control system with a sEMG signal as the control signal is still effective when the training load and test load are not equal (inter-loads) [13]. Our study, which is shown in Table 2, suggests that when the training set data and the testing set data for the BPNNs are from the same loads (intra-load), the accuracy of gait recognition at each speed is far superior to the accuracy for when the data is from different loads (inter-loads) $(88.6-65.11 \%$ at V3, $94.25-71.2 \%$ at V5, 92.56-71.96\% at V7, and total accuracy $91.81-69.42 \%$, respectively). This further confirms that load variation has a significant effect on gait recognition using a sEMG signal. Therefore, it is safe to say that an exoskeleton (or prosthesis) control system that is trained in a single load is not sufficient in the case of multi-load applications.

In addition, in order to further study the effect of load variation on gait recognition accuracy in real-time environments and the possibility of improving gait recognition at the load level, we polled all the data from the four loads as a testing set at each speed to simulate a varied load environment, and we randomly combined the four kinds of data into 15 data sets as the training set to simulate the possible loads that people face in real-time environments. Our results at three different speeds showed the same conclusion: when the training data is from more types of loads, the gait recognition is more accurate as shown in Figure 6. The statistical analysis shows that the number of the kinds of loads in the training set has a substantial influence on the accuracy of gait recognition $(p<0.001)$, which implies that this is an effective way to improve the accuracy of gait recognition, i.e., by increasing the data from more types of loads in the training set.

In our study, we noted that the speed has a significant impact on the accuracy of gait recognition, as we show in Table 1. The total average gait recognition accuracies from the four loads under three speeds are obviously different. Of the four accuracies, the highest value appears at V5 (94.25\%), followed by the value at V7 (92.55\%), and the lowest value (88.61\%) appears at V3. The result is in accordance with Rossi et al. [22], whose study revealed that the performance with fast gaits is much better than that in slower gaits. Many researchers have addressed the effect of speed on gait. Gabel et al. [37] analyzed the relationship between the speed and the characteristics of the myoelectric signal, and concluded that a natural walking speed can lead to an ideal average sEMG. Kadaba et al. [38] revealed that a normal comfortable pace is conducive to improving the repeatability of the EMG signal during the gait cycle. This may also explain to a certain extent why the gait recognition accuracy at $\mathrm{V} 5$ is the highest, because V5 $(5 \mathrm{~km} / \mathrm{h})$ is the most commonly used walking speed in daily life. As for why the gait recognition accuracy is the lowest at $\mathrm{V} 3$, a possible reason may be that $\mathrm{V} 3$ is far below the normal walking speed. Due to the slow speed, people are in a relaxed and casual state in the experiment. Even though the speed of the treadmill is uniform at V3, the speeds of the subjects fluctuate greatly and the stride cannot be kept constant, and so there are great differences in muscle activity while walking [39]. The muscle force of the target muscle cannot maintain an ideal isometric state [40], and the nervous system activity is also unstable [17], which leads to a poor result in gait recognition. Compared to V3, the subject more easily maintains a constant pace and speed on a constant-speed treadmill at V7, so that the muscle action and sEMG signal have better stability, which may be the reason for why this results in a higher accuracy of gait recognition than that at V3. In addition, V7 is faster than the other two speeds so that the muscles at V7 fatigue more easily after a period of walking under the same trial conditions [41], which reduces the accuracy of gait recognition at V7.

However, there are some drawbacks to our experiment. For example, we selected healthy people as subjects, who were not really typical of the users of exoskeletons (stroke patients and lower limb 
hemiplegia patients). Second, we chose only adult males as subjects in the experiment, and we did not consider the effect of gender and age on gait recognition, which does have a significant effect on muscle activity $[42,43]$ and gait parameters [44,45]. Thus, future studies will mainly focus on the following two aspects: first, studying subjects who are closer to those who use exoskeletons. Second, more categories of subjects (different age, different gender, and even different regions and races) will be considered to improve the effectiveness of the lower-limb exoskeleton and the prostheses control system in real-time environments.

\section{Conclusions}

This paper mainly demonstrates that the load variation can significantly affect the accuracy of gait recognition. At the same time, a much higher accuracy in gait recognition was found when the BPNN data was taken from an intra-load than when the data was taken from inter-loads. Furthermore, we arbitrarily combined the data from the four loads at each speed to simulate possible loads that the exoskeleton (or prosthesis) would face in real-time environments. The results reveal that when the training set data is taken from more types of loads, a better level of performance is achieved in gait recognition. This implies that an exoskeleton (or prosthesis) control system that is trained in a single load or part of a load does not work well in the face of multi-load applications, and that an effective method of improving the performance is to add training set data from more types of loads. The results could be useful, not only in exoskeleton or prosthesis myoelectric guidance, but also in complementing evidence showing that loads that heavily influence lower limb muscular activity can be useful for deepening the extent by which muscular variability occurs during walking, which appears to be related not only to walking speed, but also to gender and age.

Author Contributions: Conceptualization, X.Z; methodology, Z.T.; data curation, C.L.; writing- original draft preparation, X.Z.; project administration, S.S.

Funding: This research was funded by the National Natural Science Foundation of China (No. 61562072), the Key Research Plan of Jiangxi Province (No. 20161BBE50058) and the National Natural Science Foundation of China major research program (No. 91748127).

Conflicts of Interest: The authors declare no conflict of interest.

\section{References}

1. Johansson, J.L.; Sherrill, D.M.; Riley, P.O.; Bonato, P.; Herr, H. A clinical comparison of variable-damping and mechanically passive prosthetic knee devices. Am. J. Phys. Med. Rehabil. 2005, 84, 563-575. [CrossRef] [PubMed]

2. Marchal-Crespo, L.; Reinkensmeyer, D.J. Review of control strategies for robotic movement training after neurologic injury. J. Neuroeng. Rehabil. 2009, 6, 20:1-20:15. [CrossRef] [PubMed]

3. Riener, R.; Lunenburger, L.; Jezernik, S.; Anderschitz, M.; Colombo, G.; Dietz, V. Patient-cooperative strategies for robot-aided treadmill training: first experimental results. IEEE Trans. Neural. Syst. Rehabil. Eng. 2005, 13, 380-394. [CrossRef] [PubMed]

4. Li, Y.; Gao, F.; Chen, H.; Xu, M. Gait recognition based on EMG with different individuals and sample sizes. In Proceedings of the 2016 35th Chinese Control Conference (CCC), Chengdu, China, 27-29 July 2016; pp. 4068-4072. [CrossRef]

5. Joshi, C.D.; Lahiri, U.; Thakor, N.V. Classification of gait phases from lower limb EMG: Application to exoskeleton orthosis. In Proceedings of the 2013 IEEE Point-of-Care Healthcare Technologies (PHT), Bangalore, India, 16-18 January 2013; pp. 228-231. [CrossRef]

6. Kong, K.; Tomizuka, M. Smooth and continuous human gait phase detection based on foot pressure patterns. In Proceedings of the 2008 IEEE International Conference on Robotics and Automation, Pasadena, CA, USA, 19-23 May 2008; pp. 3678-3683. [CrossRef]

7. Oskoei, M.A.; Hu, H. Myoelectric control systems-A survey. Biomed. Signal Process. Control 2007, 2, $275-294$. [CrossRef] 
8. Sang, W.L.; Yi, T.; Han, J.-S.; Jang, H.; Kim, H.-H.; Jung, J.-W.; Bien, Z. Walking phase recognition for people with lower limb disability. In Proceedings of the 2007 IEEE 10th International Conference on Rehabilitation Robotics, Noordwijk, The Netherlands, 13-15 June 2007; pp. 60-67. [CrossRef]

9. Yang, P.; Chen, L.; Guo, X.; Wang, X.; Li, L. Artificial Lower Limb with Myoelectrical Control Based on Support Vector Machine. In Proceedings of the 6th World Congress on Intelligent Control and Automation, Dalian, China, 21-23 June 2006; Volume 2, pp. 9486-9489. [CrossRef]

10. Wu, J.; Wu, Q.; Sun, S. Research on classification algorithm of reduced support vector machine for low limb movement recognition. China Mech. Eng. 2011, 22, 433-438.

11. Scheme, E.; Fougner, A.; Stavdahl, Ø.; Chan, A.C.; Englehart, K. Examining the adverse effects of limb position on pattern recognition based myoelectric control. In Proceedings of the 2010 Annual International Conference of the IEEE Engineering in Medicine and Biology, Buenos Aires, Argentina, 31 August-4 September 2010; pp. 6337-6340. [CrossRef]

12. Lee, S.W.; Yi, T.; Jung, J.-W.; Bien, Z. Design of a gait phase recognition system that can cope with EMG electrode location variation. IEEE Trans. Autom. Sci. Eng. 2017, 14, 1429-1439. [CrossRef]

13. Lorrain, T.; Jiang, N.; Farina, D. Influence of the training set on the accuracy of surface EMG classification in dynamic contractions for the control of multifunction prostheses. J. Neuroeng. Rehabil. 2011, 8, 25:1-25:8. [CrossRef] [PubMed]

14. Young, A.J.; Hargrove, L.J.; Kuiken, T.A. Improving myoelectric pattern recognition robustness to electrode shift by changing interelectrode distance and electrode configuration. IEEE Trans. Biomed. Eng. 2012, 59, 645-652. [CrossRef] [PubMed]

15. Al-Timemy, A.H.; Bugmann, G.; Escudero, J.; Outram, N. A preliminary investigation of the effect of force variation for myoelectric control of hand prosthesis. In Proceedings of the 2013 35th Annual International Conference of the IEEE Engineering in Medicine and Biology Society (EMBC), Osaka, Japan, 3-7 July 2013; pp. 5758-5761. [CrossRef]

16. Scheme, E.; Englehart, K. Electromyogram pattern recognition for control of powered upper-limb prostheses: State of the art and challenges for clinical use. J. Rehabil. Res. Dev. 2011, 48, 643-659. [CrossRef] [PubMed]

17. Tang, Z.; Yu, H.; Cang, S. Impact of load variation on joint angle estimation from surface EMG signals. IEEE Trans. Neural. Syst. Rehabil. Eng. 2015, 24, 1342-1350. [CrossRef] [PubMed]

18. He, H.; Kiguchi, K. A study on EMG-based control of exoskeleton robots for human lower-limb motion assist. In Proceedings of the 6th IEEE International Special Topic Conference on Information Technology Applications in Biomedicine, Tokyo, Japan, 8-11 November 2007; pp. 292-295. [CrossRef]

19. Tang, Z.; Sun, S.; Wang, J.; Zhang, K. An ergonomics evaluation of the vibration backpack harness system in walking. Int. J. Ind. Ergonom. 2014, 44, 753-760. [CrossRef]

20. Disselhorst-Klug, C.; Schmitz-Rode, T.; Rau, G. Surface electromyography and muscle force: Limits in sEMG-force relationship and new approaches for applications. Clin. Biomech. 2009, 24, 225-235. [CrossRef] [PubMed]

21. Charteris, J. Comparison of the effects of backpack loading and of walking speed on foot-floor contact patterns. Ergonomics 1998, 41, 1792-1809. [CrossRef] [PubMed]

22. De Rossi, S.M.M.; Crea, S.; Donati, M.; Reberšek, P.; Novak, D.; Vitiello, N.; Carrozza, M.C. Gait segmentation using bipedal foot pressure patterns. In Proceedings of the 2012 4th IEEE RAS \& EMBS International Conference on Biomedical Robotics and Biomechatronics (BioRob), Rome, Italy, 24-27 June 2012; pp. 361-366. [CrossRef]

23. Dahl, K.D.; Wang, H.; Popp, J.K.; Dickin, D.C. Load distribution and postural changes in young adults when wearing a traditional backpack versus the BackTpack. Gait Posture 2016, 45, 90-96. [CrossRef] [PubMed]

24. Pascoe, D.D.; Pascoe, D.E.; Wang, Y.T.; Shim, D.-M.; Kim, C.K. Influence of carrying book bags on gait cycle and posture of youths. Ergonomics 1997, 40, 631-640. [CrossRef] [PubMed]

25. Ghoussayni, S.; Stevens, C.; Durham, S.; Ewins, D. Assessment and validation of a simple automated method for the detection of gait events and intervals. Gait Posture 2004, 20, 266-272. [CrossRef] [PubMed]

26. Meng, M.; Luo, Z.; She, Q.; Ma, Y. Automatic recognition of gait mode from EMG signals of lower limb. In Proceedings of the 2010 2nd International Conference on Industrial Mechatronics and Automation (ICIMA 2010), Wuhan, China, 30-31 May 2010; Volume 1, pp. 282-285. [CrossRef]

27. Hudgins, B.; Parker, P.; Scott, R.N. A new strategy for multifunction myoelectric control. IEEE Trans. Biomed. Eng. 1993, 40, 82-94. [CrossRef] [PubMed] 
28. Phinyomark, A.; Phukpattaranont, P.; Limsakul, C. Feature reduction and selection for EMG signal classification. Expert Syst. Appl. 2012, 39, 7420-7431. [CrossRef]

29. Veer, K.; Sharma, T. A novel feature extraction for robust EMG pattern recognition. J. Med. Eng. Technol. 2016, 40, 149-154. [CrossRef] [PubMed]

30. Zhang, F.; Li, P.; Hou, Z.-G.; Lu, Z.; Chen, Y.; Li, Q.; Tan, M. sEMG-based continuous estimation of joint angles of human legs by using BP neural network. Neurocomputing 2012, 78, 139-148. [CrossRef]

31. Oskoei, M.A.; Hu, H. Support vector machine-based classification scheme for myoelectric control applied to upper limb. IEEE Trans. Biomed. Eng. 2008, 55, 1956-1965. [CrossRef] [PubMed]

32. Alkan, A.; Günay, M. Identification of EMG signals using discriminant analysis and SVM classifier. Expert Syst. Appl. 2012, 39, 44-47. [CrossRef]

33. Kim, K.S.; Choi, H.H.; Moon, C.S.; Mun, C.W. Comparison of k-nearest neighbor, quadratic discriminant and linear discriminant analysis in classification of electromyogram signals based on the wrist-motion directions. Curr. Appl. Phys. 2011, 11, 740-745. [CrossRef]

34. Kim, S.-G.; Nam, C.-W.; Yong, M.-S. The effect of increase in baggage weight on elderly women's lower extremity muscle activation during gait. Arch. Gerontol. Geriatr. 2014, 59, 574-576. [CrossRef] [PubMed]

35. Simpson, K.M.; Munro, B.J.; Steele, J.R. Backpack load affects lower limb muscle activity patterns of female hikers during prolonged load carriage. J. Electromyogr. Kinesiol. 2011, 21, 782-788. [CrossRef] [PubMed]

36. Catalfamo, P.; Acevedo, R.; Ghoussayni, S.; Ewins, D. Comparison of kinematic and pressure measurement reference methods used in gait event detection. Footwear Sci. 2014, 6, 193-202. [CrossRef]

37. Gabel, R.H.; Brand, R.A. The effects of signal conditioning on the statistical analyses of gait EMG. Clin. Neurophysiol. 1994, 93, 188-201. [CrossRef]

38. Kadaba, M.P.; Ramakrishnan, H.K.; Wootten, M.E.; Gainey, J.; Gorton, G.; Cochran, G.V.B. Repeatability of kinematic, kinetic, and electromyographic data in normal adult gait. J. Orthop. Res. 1989, 7, 849-860. [CrossRef] [PubMed]

39. Di Nardo, F.; Mengarelli, A.; Maranesi, E.; Burattini, L.; Fioretti, S. Gender differences in the myoelectric activity of lower limb muscles in young healthy subjects during walking. Biomed. Signal Process. Contol 2015, 19, 14-22. [CrossRef]

40. Tang, Z.; Zhang, K.; Sun, S.; Gao, Z.; Zhang, L.; Yang, Z. An upper-limb power-assist exoskeleton using proportional myoelectric control. Sensors 2014, 14, 6677-6694. [CrossRef] [PubMed]

41. Den Otter, A.R.; Geurts, A.C.H.; Mulder, T.; Duysens, J. Speed related changes in muscle activity from normal to very slow walking speeds. Gait Posture 2004, 19, 270-278. [CrossRef]

42. Chiu, M.-C.; Wang, M.-J. The effect of gait speed and gender on perceived exertion, muscle activity, joint motion of lower extremity, ground reaction force and heart rate during normal walking. Gait Posture 2007, 25, 385-392. [CrossRef] [PubMed]

43. Mengarelli, A.; Maranesi, E.; Burattini, L.; Fioretti, S.; Di Nardo, F. Co-contraction activity of ankle muscles during walking: A gender comparison. Biomed. Signal Process. Control 2017, 33, 1-9. [CrossRef]

44. Chung, M.-J.; Wang, M.-J.J. The change of gait parameters during walking at different percentage of preferred walking speed for healthy adults aged 20-60 years. Gait Posture 2010, 31, 131-135. [CrossRef] [PubMed]

45. Di Nardo, F.; Laureati, G.; Strazza, A.; Mengarelli, A.; Burattini, L.; Agostini, V.; Nascimbeni, A.; Knaflitz, M.; Fioretti, S. Is child walking conditioned by gender? Surface EMG patterns in female and male children. Gait Posture 2017, 53, 254-259. [CrossRef] [PubMed]

(C) 2018 by the authors. Licensee MDPI, Basel, Switzerland. This article is an open access article distributed under the terms and conditions of the Creative Commons Attribution (CC BY) license (http://creativecommons.org/licenses/by/4.0/). 